\title{
A Lattice Boltzmann Scheme for Semiconductor Dynamics
}

\author{
S. SUCCI and P. VERGARI \\ IBM European Center for Scientific and Engineering Computing, v. Oceano Pacifico 171, Rome, 00144, Italy \\ Mathematics Department, University of Catania, via Andrea Doria, Catania, Italy
}

We discuss an extension of the Lattice Boltzmann method which may prove useful for the numerical study of electron transport in semiconductors.

Keywords: semiconductor, discrete kinetic theory

\section{INTRODUCTION}

Lattice-gas (LG) models obeying cellular automata rules and the related Lattice-Boltzmann (LB) technique have known a rapid expansion in the recent years mostly in connection with the simulation of complex hydrodynamic phenomena $[1,2,3,4]$ The driving force behind these methods is their outstanding amenability to parallel processing which stems from the extreme space-time locality of cellular automata rules. So far, LG and LB methods have been directed chiefly to the investigation of hydrodynamic problems. However, in the recent years, extensions of the LB method have been developed which are capable of handling several generalizations of the NavierStokes equations, including transport of passive scalars, and flows with thermal and chemical effects. Precisely in this spirit of "generalized" hydrodynamics, we present here an extension of the LB model which accounts for the generalized hydrodynamic equations describing the motion of hot electrons in semiconductors.

\section{THE LATTICE BOLTZMANN EQUATION}

The Lattice Boltzmann (LB) method is based on the following finite-difference kinetic equation [5]:

$N_{i}\left(x_{k}+c_{i k}, t+1\right)-N_{i}(x, t)=\sum_{j=1}^{b} \Omega_{i j}\left(N_{j}-N_{j}^{e q}\right)$

where $N_{i}$ represent particle populations moving along the direction identified by the discrete speed $c_{i k}, i$ denoting the propagation direction and $k$ runs over the spatial dimension. $\Omega_{i j}$ is the scattering matrix mediating two-body collisions between state i and $\mathrm{j}$ and $N_{j}^{e q}$ are local equilibrium populations. The large-scale limit of the equation (1) is shown to converge to the Navier-Stokes equations of fluid dynamics, provided the following mass and momentum conservation laws are fulfilled:

$$
\sum_{i=1}^{b} \Omega_{i j}=0, \quad \sum_{i=1}^{b} \Omega_{i j} c_{i k}=0
$$

In addition, in order to recover isotropy at the macroscopic level, the set of discrete speeds must ensure isotropy of the fourth order tensor $T_{i j k l}=c_{i} c_{j} c_{k} c_{l}$.

This latter condition places rather restrictive requirements on the class of lattices suitable to hydrodynamic purposes. 
The derivation of the Navier-Stokes equations formally proceeds by contracting over the discrete speeds degrees of freedom so as to generate evolution equation for the moments of the discrete distribution function $N_{i}$. These equations are then closed via the usual assumption of weak departures from smoothly varying thermodynamic equilibria. A sensible choice of the local equilibrium distribution function is also instrumental to achieve the correct hydrodynamic equations. A suitable choice is a local maxwellian expanded to 2nd order in the Mach number:

$$
N_{i}^{e q}=\frac{\rho}{b}\left(1+2 c_{i k} M_{k}+\frac{1}{2} Q_{i k l} M_{k} M_{l}\right)
$$

where $M_{k} \equiv u_{k} / c_{s}, c_{s}$ being the sound speed and $Q_{i k l}=$ $c_{i k} c_{i l}-c_{s}^{2} \delta_{k l}$ is the projector along the $i$-th propagation direction.

On the analytical side one can prove a global $\mathrm{H}$ theorem which guarantees numerical stability in the linear regime (flow speed $u \ll c_{s}$ ) provided the spectrum of $\Omega_{i j}$ be confined within the strip $-2<\lambda<0$.

\section{HYDRODYNAMIC SEMICONDUCTOR EQUATIONS (HSE)}

In the recent years, hydrodynamical models $[6,7,8]$ describing carrier flow in semiconductor devices have attracted considerable attention as an alternative tool to kinetic theory to describe carrier flow in semiconductor devices. In this paper, we shall restrict our attention to the hydrodynamic model proposed by Anile and coworkers [9].

The one-dimensional field equations read as follows

$$
\begin{gathered}
\partial_{t} \rho+\partial_{x}(\rho u)=0 \\
\partial_{t} \rho u+\partial_{x}\left(\rho u^{2}\right)=-e \rho E-\partial(\rho T)-J / \tau_{p}+\partial_{x} v \partial_{x} \rho u \\
\partial_{t} W+\partial_{x}(W u)=-e \rho u E-\partial(\rho u T)+ \\
\partial_{x} K \partial_{x} T+\left(\frac{3}{2} \rho T_{0}-W\right) / \tau_{w}
\end{gathered}
$$

where $\rho$ is the carrier mass density, $u$ the mean flow speed and $W$ is the total energy per unit volume.
In the following, we shall deal with the problem of finding an appropriate LB scheme yielding the eqs. (4-6) in the large-scale limit.

\section{GENERALIZED HYDRODYNAMIC EQUATIONS (GHE)}

Since the GHE's do involve energy as a dynamic variable, the corresponding LB scheme must necessarily cater for at least two energy levels $\varepsilon_{i}=m_{i} c_{i}^{2}$. The minimal choice is the following 4-state (2-energy) discrete-speed scheme:

$$
\begin{array}{cll}
c_{i}= \pm 1, \quad m_{i}=1, & i=1,2 \quad\left(\varepsilon_{i}=1\right) \\
c_{i}= \pm 2, & m_{i}=m, \quad i=3,4 \quad\left(\varepsilon_{i}=4 m\right)
\end{array}
$$

This discrete set gives rise to the following definition of temperature

$$
T=\frac{\rho_{1}}{\rho} c_{1}^{2}+\frac{\rho_{2}}{\rho} c_{2}^{2}
$$

where $\rho_{1}=\left(N_{1}+N_{2}\right), \rho_{2}=\left(N_{3}+N_{4}\right) m$. This results in a dynamical range of temperatures between $T=1$ and $T=4$ corresponding to full occupation of the lower(higher) energy levels respectively. The equilibrium temperature lies in between and depends on the mass ratio $m$ according to $T^{e}=\frac{1+4 m}{1+m}$

This set generates a $4 \times 4$ collision matrix by six distinct matrix elements $a_{1}, a_{2}, b_{1}, b_{2}, c, d$

$$
\Omega_{i j}=\left[\begin{array}{cccc}
a_{1} & b_{1} & c & d \\
b_{1} & a_{1} & d & c \\
c & d & a_{2} & b_{2} \\
d & c & b_{2} & a_{2}
\end{array}\right]
$$

Clearly $a_{i}, b_{i}$ describe "elastic" collisions among particles with the same energy, while $c, d$ are associated with "inelastic" cross-energy collisions. The latter are responsible for temperature evolution since they change $\rho_{1}, \rho_{2}$ separately, while leaving the total density $\rho=\rho_{1}+\rho_{2}$ unchanged. The next step is to identify the eigenvectors values of the collision matrix which generate the hydrodynamic fields $\rho, J, W$ in compliance with the GHE's. Using the mass ratio $m$ as a free parameter, some algebra yields the following 
orthogonal set of eigenvectors (scalar products being weighted with the array $m_{i}=(1,1, m, m)$.

$$
\begin{gathered}
V_{i}^{(1)}=1_{i}=(1,1,1,1) ; V_{i}^{(2)}=c_{i}=(1,-1,2,-2) ; \\
V_{i}^{(3)}=c_{i}^{2}-2=(-1,-1,2,2) ; V_{i}^{(4)}= \\
c_{i}\left(c_{i}^{2}-3\right)=(-2,2,2,-2)
\end{gathered}
$$

corresponding to the specific choice $m=\frac{1}{2}$ yielding $T^{e}=c_{s}^{2}=2$.

Straightforward projection upon the four eigenvectors yields:

$$
\begin{gathered}
\partial_{t} \rho+\partial_{x} \rho u=0 \\
\partial_{t} \rho u+\partial_{x} S=-2 \partial_{x} \rho \\
\partial_{t} S+\partial_{x} h=-2 \partial_{x} J+\lambda_{3}\left(S-S^{e}\right) \\
\partial_{t} h+2 \partial_{x} S=\lambda_{4}\left(h-h^{e}\right)
\end{gathered}
$$

where:

$$
\begin{aligned}
& \rho=\sum_{i} m_{i} N_{i} V_{i}^{(1)}, J=\sum_{i} m_{i} N_{i} V_{i}^{(2)}, \\
& \quad S=\sum_{i} m_{i} N_{i} V_{i}^{(3)}, h=\sum_{i} m_{i} N_{i} V_{i}^{(4)} .
\end{aligned}
$$

According to standard practice, we now make an adiabatic assumption on both the deviatoric stress tensor $S$ and the heat-like flux $h$ :

$$
\begin{gathered}
S \simeq S^{e}+\lambda_{3}^{-1} \partial_{x} J+\lambda_{4}^{-1} \partial_{x} h \\
h \simeq h^{e}+2 \lambda_{4}^{-1} \partial_{x} S
\end{gathered}
$$

Since fluctuations do not contribute to conserved hydrodynamic fields, one has $\rho=\rho^{e}, J=J^{e}, q^{e}=0$, which yields $S^{e}=\rho u^{2}+\rho\left(T-T_{e}\right), T^{e}=2$.

By plugging eq.(26) into (23), the momentum equation reads

$$
\partial_{t} \rho u+\partial_{x} \rho u^{2}=-\partial_{x} \rho T-\lambda_{3}^{-1} \partial_{x}^{2}(\rho u)
$$

This is exactly the Navier-Stokes equation for a onedimensional fluid with viscosity $v=-\lambda-1$

As to the energy equation, by using (27) and (25) we obtain

$$
\begin{aligned}
\partial_{t} \rho W+\partial_{x} \rho W=-2 \partial_{x} \rho u T & +2 \lambda_{4}^{-1} \partial_{x}^{2}\left(W-\frac{\rho T^{e}}{2}\right) \\
& +\frac{\lambda_{3}}{2}\left(W-\rho u^{2}-\rho T^{e}\right)
\end{aligned}
$$

where $T^{\prime} \equiv T-T^{e}$ is the temperature fluctuation.

This is "almost" the correct energy equation, with a Baccarani-Wordeman type of collisional relaxation, a detailed definition of "almost" to be clarified in the sequel.

Let us now discuss how to model the effects specific to the physics of semiconductors: the presence of an external electric field and collisional relaxation.

Electric field effects are readily incorporated by adding a suitable forcing term $F_{i}$ to the rhs of eq.(1).

This term must deposit $\dot{J}_{E}=-e \rho E$ units of momentum and $u \dot{J}_{E}$ units of energy per unit volume of the flow. Straightforward decomposition of the forcing term $F_{i}$ onto the four eigenvectors defined in yields the desired expression:

$$
\begin{gathered}
F_{i}=\frac{\dot{J}_{E} c_{i}}{M_{1}}+\frac{u \dot{J}_{E}\left(c_{i}^{2}-2\right)}{M_{2}} \\
M_{1}=\sum_{i} m_{i} c_{i}^{2}=6 ; M_{2}=\sum_{i} m_{i} Q_{i}^{2}=2
\end{gathered}
$$

A similar trick can also be applied to mimic the effects of the collisional term with a relaxation term of the form

$$
G_{i}=-\left(N_{i}-N_{i}^{0}\right) / \tau
$$

where $N_{i}^{0}$ is a zero-flow (global) equilibrium distribution function.

This yields a momentum relaxation $-J / \tau$ and an energy relaxation $\left(W-W_{0}\right) / \tau$ where $W_{0}=\rho T_{e} / 2$. The final energy equation reads now as follows

$$
\begin{array}{r}
\partial_{t} W+\partial_{x} u W=-\partial_{x} \rho u T+\lambda_{4}^{-1} \partial_{x}^{2}\left(W-\frac{\rho T^{e}}{2}\right)+ \\
\frac{\lambda_{3}}{2}\left(W-\rho u^{2}-\rho T^{e}\right)-\rho e E u
\end{array}
$$

This is exactly the GHE energy equation with $K=-\frac{\lambda_{3}^{-1}}{2}$ provided the following conditions are met:
1.
$\partial_{x}^{2} \rho \ll \partial_{x}^{2} W$
2.
$u^{2} \ll T^{e}=2$
3.
$\lambda_{3} \tau=-1$ 
The first two conditions both subsume the same assumption of weak-compressibility which lies at very heart of the LBE theory. In this respect, they don't set any further limitation beyond those currently accepted for usual fluid dynamics purposes.

The third condition restricts the applicability of the model to situations where energy and momentum relax on the same time scale, this time scale being also the one controlling the thermal conductivity.

\section{FINAL CONSIDERATIONS}

We have presented a minimal extended Lattice-Boltzmann scheme catering for the inclusion of the physical effects required to model the behaviour of hot electrons in semiconductor devices: the presence of an external electric field and local relaxation due to electron collisions with the phononic background. Owing to its minimality, the scheme doesn't allow for more than two independent time scales. Once these are chosen so as to control the momentum and energy diffusivity, no additional degrees of freedom are available to describe collisional relaxation of momentum and energy independently. This limitation shouldn't bear any fundamental character, and can possibly be lifted by enlarging the set of discrete speeds.
A more fundamental limitation, which is shared with "plain" LBE schemes, is that the model only operates for weakly compressible flows characterized by Mach numbers well below one.

In view of such a limitation the scheme might prove viable only for the description of the bulk flow dynamics, while regions of strong heterogeneity are likely to call for more sophisticated schemes involving more speeds and/or a non-uniform grid discretization. A careful investigation of this issue is currently underway.

\section{Acknowledgements}

The work was partially supported by CNR $n^{\circ} 92.00 .53801$

\section{References}

[1] U. Frisch, D. d'Humières, B. Hasslacher, P. Lallemand, Y. Pomeau, J. P. Rivet, Complex Systems 1 (1987) 649

[2] Physica D Vol. 47 (1991), n. 1-2 special issue

[3] J. Stat. Phys., 68, 3/4 (Aug. 1992), special issue

[4] U. Frisch, B. Hasslacher, and Y. Pomeau, Phys. Rev. Lett. 56 (1986) 15.

[5] R. Benzi, S. Succi, M. Vergassola, Physics Reports 222 (3) 1992, and references therein

[6] A. Forghieri, R. Guerrieri, P. Ciompoli, A.Gnudi, M.Rudan, G.Baccarani, IEEE Trans.on Comp.Aided Desig. vol.7, 1988

[7] C. Gardner, J. Jerome, D. Rose, IEEE Trans. on Comp. Aided Desig. vol.8, 1989

[8] C. Gardner. IEEE Trans. on Comp.Aided Desig. vol.38, 1991

[9] A.M. Anile, S. Pennisi, Phys. Rev. B vol. 46, n.20, 13 186, 1992. 

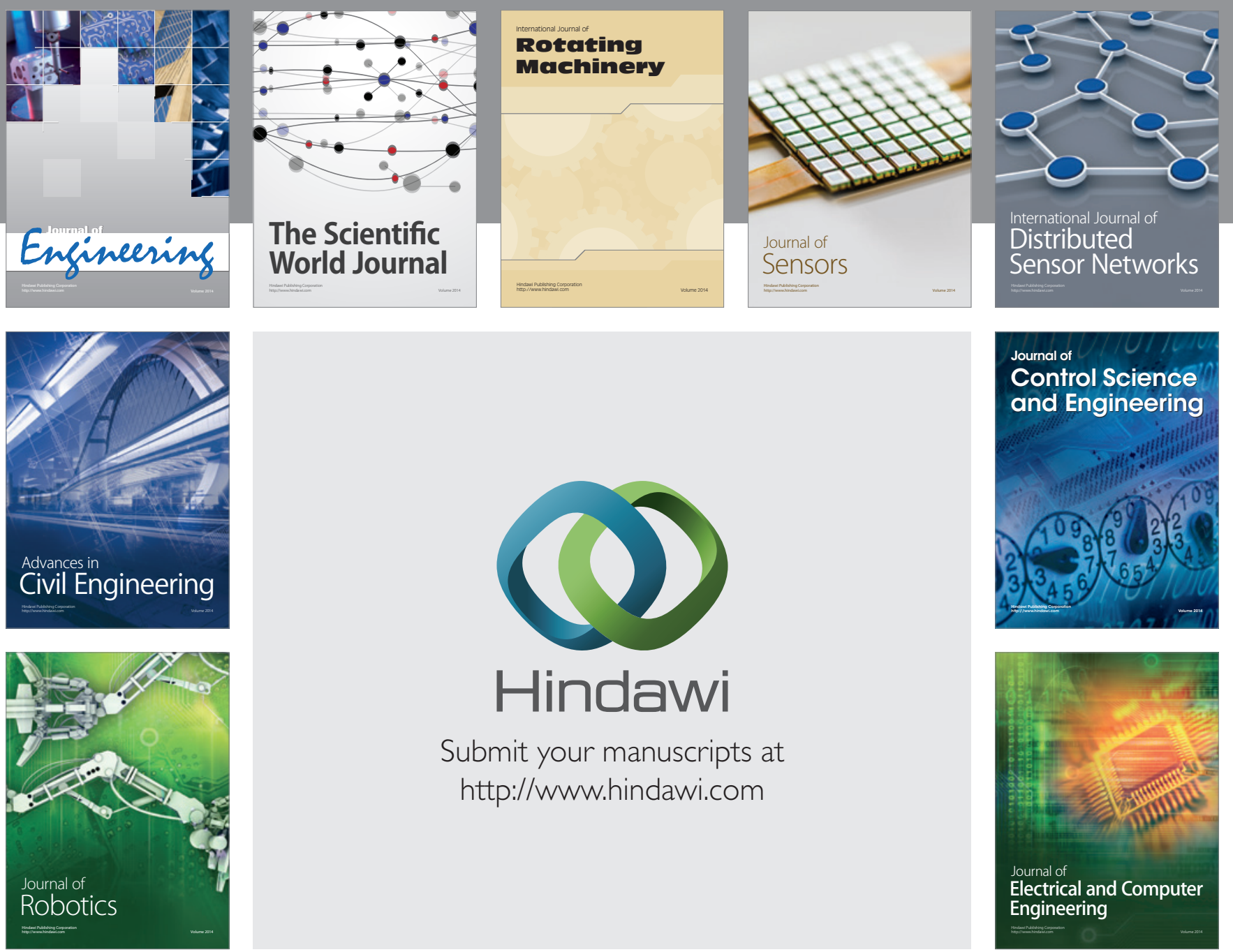

Submit your manuscripts at

http://www.hindawi.com
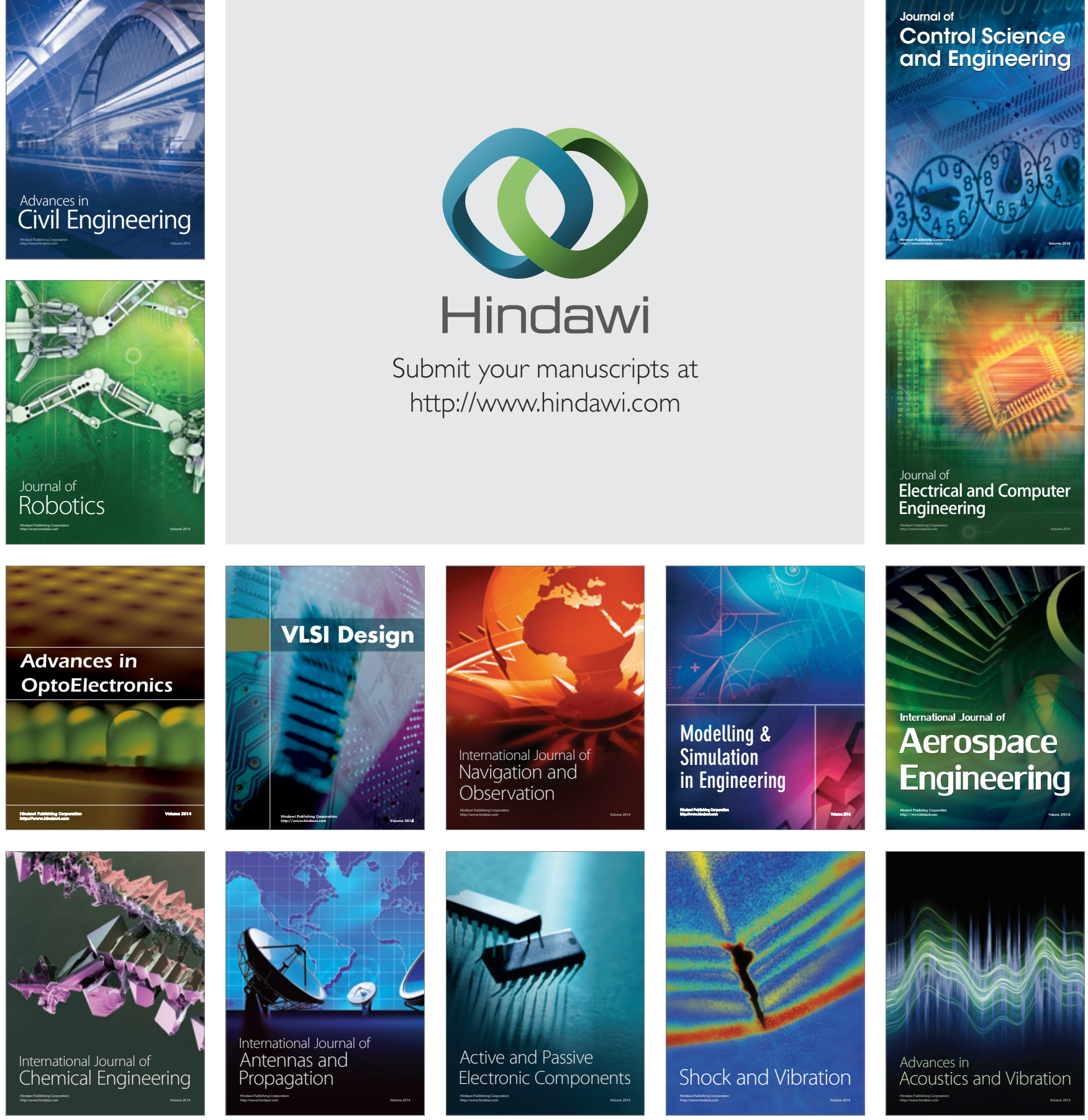